\title{
Epidermolytic Hyperkeratosis (Bullous Congenital Ichthyosiform Erythroderma) Genetic Linkage to Chromosome 12q in the Region of the Type II Keratin Gene Cluster
}

\author{
Leena Pulkkinen, Angela M. Christiano, Robert G. Knowlton, and Jouni Uitto \\ Departments of Dermatology, and Biochemistry and Molecular Biology, Jefferson Medical College, and Section of Molecular \\ Dermatology, Jefferson Institute of Molecular Medicine, Thomas Jefferson University, Philadelphia, Pennsylvania 19107
}

\begin{abstract}
Epidermolytic hyperkeratosis (EHK) is an autosomal dominant genodermatosis characterized by hyperkeratosis and blistering of the skin. Histopathology demonstrates suprabasilar blister formation with aggregation of tonofilaments. In this study, we tested the hypothesis that the EHK phenotype is linked to one of the suprabasilar keratins (KRT10 or KRT1) present in the types I and II keratin gene clusters in chromosomes $17 q$ and $12 q$, respectively. For this purpose, Southern hybridizations were performed with DNA from a large kindred with EHK, consisting of 11 affected individuals in three generations. Segregation analysis with markers flanking the keratin gene clusters demonstrated linkage $(\hat{Z}=3.61$ at $\hat{\theta}=0)$ to a locus on $12 q$, while markers on $17 q$ were excluded. These data implicate KRT1, the type II keratin expressed in suprabasilar keratinocytes, as a candidate gene in this family with EHK. ( $J$. Clin. Invest. 1993. 91:357-361.) Key words: bullous skin diseases • genetic linkage analyses • genodermatoses • keratinization disorders
\end{abstract}

\section{Introduction}

Epidermolytic hyperkeratosis (EHK $)^{1}$ is a heritable keratinization disorder with a marked tendency for blistering. The prevalence of this disorder is estimated to be $\sim 1: 200,000$. (For reviews, see references 1 and 2). The disease is present at birth or shortly thereafter, and the condition can be generalized (bullous congenital ichthyosiform erythroderma) or localized, affecting primarily the flexural areas of the skin. With time, the skin becomes hyperkeratotic and verrucous, and the blistering tendency becomes less pronounced, though it can persist throughout life. The inheritance pattern is autosomal dominant, but there is considerable heterogeneity regarding the severity of the disease, as well as evidence for variable expression within families. Histopathology of the cutaneous lesions re-

Address reprint requests to Dr. Jouni Uitto, Department of Dermatology, Jefferson Medical College, 233 South 10th Street, Room 450, Philadelphia, PA 19107.

Received for publication 29 July 1992 and in revised form 29 September 1992.

1. Abbreviation used in this paper: EHK, epidermolytic hyperkeratosis.

J. Clin. Invest.

(C) The American Society for Clinical Investigation, Inc.

$0021-9738 / 93 / 01 / 0357 / 05 \quad \$ 2.00$

Volume 91, January 1993, 357-361 veals that the tissue cleavage occurs intraepidermally through separation of abnormal suprabasilar keratinocytes, combined with focal hyperkeratosis ( 3 ).

Significant progress has recently been made in delineating the underlying mutations in various forms of epidermolysis bullosa, a group of heritable blistering diseases $(4,5)$. In particular, several forms of epidermolysis bullosa simplex have been shown to result from mutations in the keratin genes expressed in the basal keratinocytes, KRT5 and KRT14 (6-8). These candidate genes were initially suggested by characteristic aggregation of tonofilaments of the basal keratinocytes (9). Similar abnormalities have been demonstrated in the suprabasilar keratinocytes in EHK, including aggregation of tonofilaments and lysis of suprabasilar keratinocytes $(10,11)$. Based on these similarities between EHK and epidermolysis bullosa simplex, we used linkage analysis to test the hypothesis that the EHK phenotype is due to mutations in the suprabasilar keratin genes (KRT10 or KRT1) present in the types I and II keratin gene clusters on chromosomes $17 \mathrm{q}$ and $12 \mathrm{q}$, respectively.

\section{Methods}

Clinical. Peripheral blood samples were obtained from a family with clinical features of EHK (Fig. 1). The affected individuals had a history of blisters at birth with accompanying erythroderma, and in the adult individuals, thickened, verrucous skin, primarily on the flexural areas on the arms and legs was noted (Fig. 1). The blister formation was considerably more frequent during the summertime, and was induced by relatively minor trauma. All affected individuals depicted pronounced hirsutism on the extremities noticeable as early as 3 yr of age (Fig. 1).

The pattern of transmission of the phenotype was consistent with autosomal dominant inheritance (Fig. 2, top). The proband (IV-9) was a 3-yr-old female with characteristic clinical features (Fig. 1). It was of interest to note that the maternal great-grandfather (1-2) of the proband was anamnestically affected, yet his 13 siblings were free of the disease. Therefore, his phenotype may have been the result of a new mutation in this kindred.

Another interesting feature was the occurrence of retinitis pigmentosa in this family. However, careful examination of the pedigree indicated that the retinitis pigmentosa mutation did not co-segregate with the EHK phenotype. In fact, the retinitis pigmentosa mutation was inherited from the maternal grandfather of the proband (II-10), while the EHK mutation was transmitted through the maternal grandmother of the proband (II-3).

Diagnostic histopathology of the skin performed on patients III-5 and IV-8 revealed prominent hyperkeratosis, zones of compact orthokeratosis, and reticular alteration with ballooning in the upper spinous and granular layers of the epidermis (Fig. 3). The epidermal cells in the region of the blister formation were enlarged and vacuolated, and in 

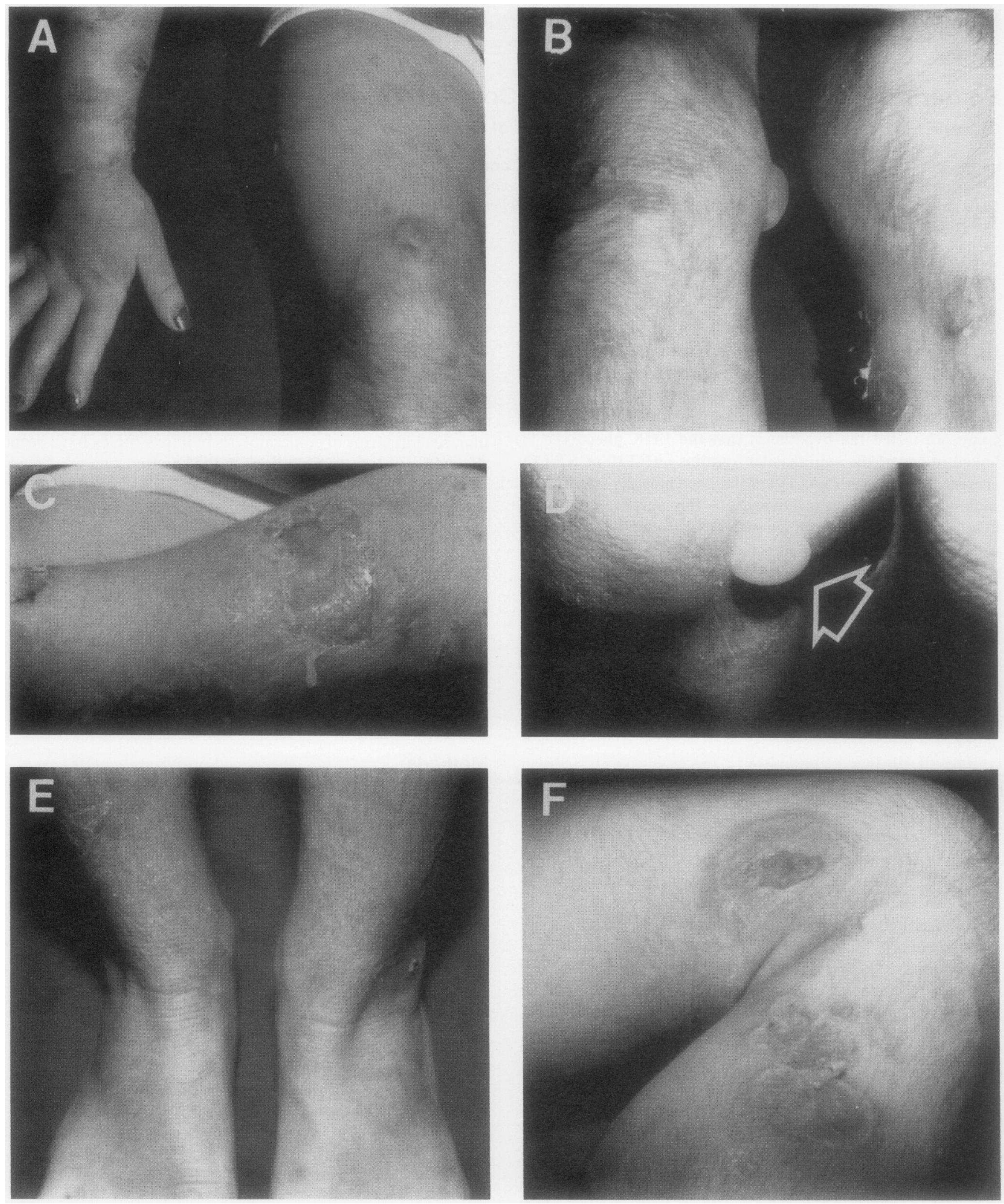

Figure 1. Clinical features of epidermolytic hyperkeratosis in the family. The proband (IV-9 in Fig. 2), a 3-yr-old female, presented with erosions and blisters primarily on the extremities. Note marked ridgelike hyperkeratosis on the knees $(A, B$, and $D)$ and on the anterior aspects of the ankles $(E)$. The presence of an intact blister of the knee is indicated $(D$, arrow), and there were extensive erosions on the arms $(A$ and $C)$ and legs $(F)$. Also, note the marked hirsutism in this young patient $(A, B$, and $F)$. 

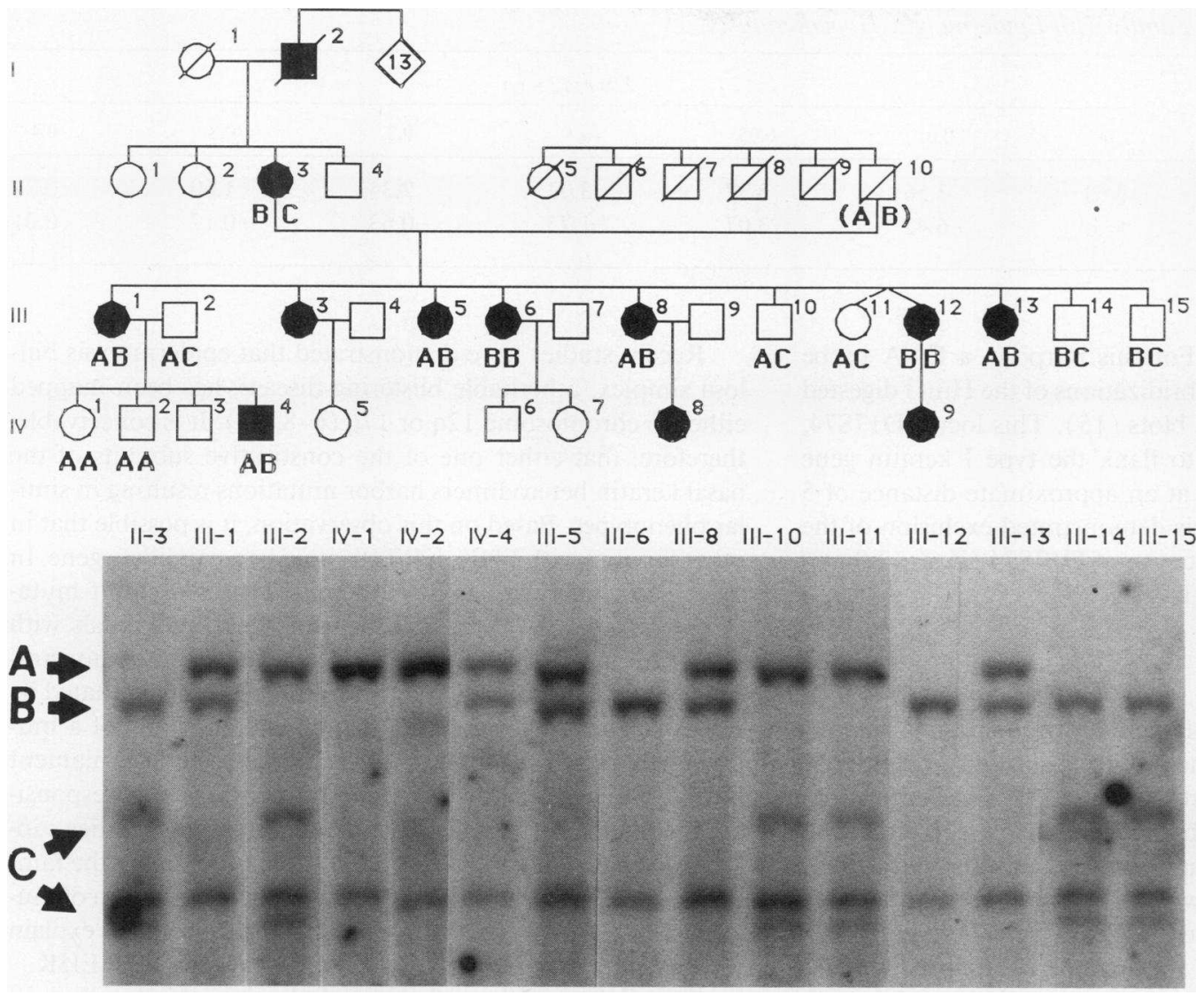

Figure 2. Pedigree of the family with epidermolytic hyperkeratosis, and Southern analysis of DNA from individuals within the family. (Top) Note the presence of EHK phenotype in 11 living individuals in three generations ( solid symbols). The genotype, as derived from Southern analysis with a COL2A1 marker, as shown in $B$, is indicated under each symbol. (Bottom) Southern blot with the WV214 probe of Hinf I digested DNA from 15 individuals identified by their pedigree number. Note the presence of three variable alleles, $A, B$, and $C$ (see text). There is a constant band between the two fragments of the $C$ allele. The results demonstrate complete co-segregation of the maternal $B$ allele with the EHK phenotype (see Table I). some areas had completely degenerated to form the blister space. These features are diagnostic for EHK (3).

DNA analysis. DNA was isolated from peripheral blood and digested either with Hinf I or MspI restriction endonuclease, according to the manufacturer's recommendations (Boehringer-Mannheim Biochemicals, Indianapolis, IN). The Southern transfer analyses of the restriction enzyme digests and probe hybridizations were performed as described previously (12). The following DNA probes were used for analysis: for chromosome 12q, pYNH15 (D12S17) (13) and WV214

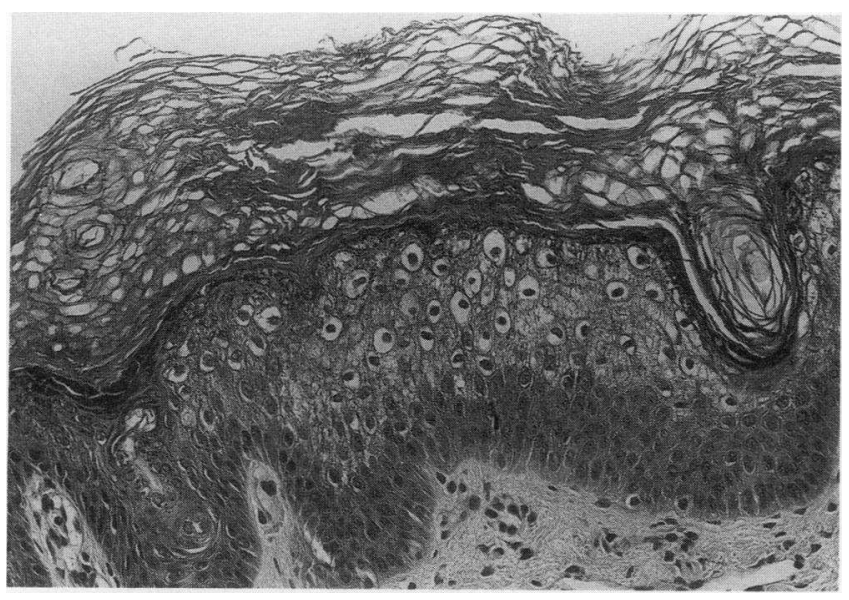

Figure 3. Histopathology of the skin in the patient (IV-8) with epidermolytic hyperkeratosis. Note prominent hyperkeratosis and hypergranulosis, and the presence of abnormal keratinocytes with ballooning degeneration in the spinous and granular layers of the epidermis.
(COL2A1) (14); for chromosome 17, pCMM86 (D17S74) (15). The DNA probes were radioactively labeled using the random primer method. Two-point linkage analyses were performed with the LIPED program (Dr. Jurgott, Columbia University, New York) (16).

\section{Results}

A large pedigree consisting of 11 living affected individuals in three generations, with clinical and histopathologic features characteristic of EHK (Figs. 1 and 2), was studied by genetic linkage analysis. To examine the possibility that the EHK phenotype in this family was linked to the type II keratin gene cluster on chromosome $12 \mathrm{q}$, an informative restriction fragment length polymorphism in COL2A1 was used to examine the co-segregation of the marker and the clinical phenotype using WV214 as a probe (Fig. 2). Southern hybridizations with DNA isolated from peripheral blood from affected and unaffected individuals, after digestion with $\mathrm{Hinf} \mathrm{I}$, revealed the presence of three alleles: A $(2.0 \mathrm{~kb}), \mathrm{B}(1.7 \mathrm{~kb})$, and C ( 1.0 and 0.7 $\mathrm{kb}$ ) (Fig. 2, bottom). The results indicated for complete co-segregation of the maternal B allele with the clinical phenotype in this family, and the maximum LOD score $(\hat{Z})$ was 3.61 at recombination fraction $(\hat{\theta}) 0.0$ ( Table $I$ ). This logarithm of the odds score establishes linkage of EHK to the region of chromosome $12 \mathrm{q}$ containing the type II keratin gene cluster $(12,17$, 18). Another RFLP in the same region, detected by the DNA probe YNH15 (D12S17), was noninformative.

The predominant type II keratin expressed in suprabasilar keratinocytes, KRT1, pairs with a type I keratin, KRT10, to form a heterodimer (19). In concurrent studies, we tested for linkage of EHK in this family to the region of $17 \mathrm{q}$ containing 


\begin{tabular}{llrrrrrrr}
\hline & & \multicolumn{7}{c}{$Z$ at $\theta\left(\theta_{\mathrm{m}}=\theta_{\mathrm{f}}\right)$} \\
\cline { 3 - 8 } Probe & Locus & 0 & \multicolumn{1}{c}{0.01} & 0.05 & 0.1 & 0.2 & 0.3 & 0.4 \\
\hline WV214 & COL2A1 & 3.61 & 3.56 & 3.32 & 3.02 & 2.35 & 1.60 & 0.74 \\
CMM86 & D17S74 & $-\infty$ & -6.42 & -3.07 & -1.75 & -0.63 & -0.17 & -0.01 \\
& & & & & & & & \\
\hline
\end{tabular}

type I keratin gene cluster. For this purpose, a DNA probe CMM86 was also used for hybridizations of the Hinf I digested DNA on the same Southern blots (15). This locus, D17S74, has previously been shown to flank the type I keratin gene cluster on chromosome $17 \mathrm{q}$ at an approximate distance of 5 cM (20). The linkage analysis demonstrated exclusion of the EHK phenotype from the vicinity of D17S74 $(Z \leq-2.0$ at $\theta$ $<0.09$, or $\sim 10 \mathrm{cM}$ ) (Table I).

\section{Discussion}

Keratins form intermediate filaments of the epidermal keratinocytes by formation of heterodimers which polymerize through discrete molecular interactions (19). The keratins can be divided into two major subgroups on the basis of molecular charge: The type II keratins are basic proteins and include the epidermal keratins 5 and 1, which are expressed in the basilar and suprabasilar keratinocytes, respectively. The type I keratins are acidic polypeptides, and include keratins 14 and 10, again expressed in the basilar and suprabasilar keratinocytes, respectively. Since the intraepidermal tissue separation observed in EHK occurs at the suprabasilar level, the keratins 1 and 10 were considered as candidate genes in EHK. Other candidate genes would include those involved in the terminal differentiation of the epidermis, such as the envelope protein filaggrin, which has been mapped to chromosome lq (21). In addition, the gene for epidermal transglutaminase on chromosome 14 could serve as a candidate gene in keratinization disorders, since this enzyme catalyzes the intermolecular crosslinking of keratins necessary for proper stratification of the skin (22). The results of our study clearly demonstrate linkage to the region containing the type II keratin gene cluster on chromosome 12q. This conclusion was based on complete co-segregation of the clinical phenotype and the COL2A1 marker which has been mapped to the locus $12 q 13.1$ (17). Although the relative map positions of COL $2 \mathrm{~A} 1$ and the type II keratin locus are not precisely established, cytogenetic and linkage data from other families indicate close proximity $(12,17,18,23)$. Our studies also excluded the region containing the type I keratin gene cluster on $17 \mathrm{q}$. Thus, the most likely candidate gene for the mutation in EHK in this family is KRT1. It should be noted that chromosome $12 \mathrm{q}$ also contains the gene locus for retinoic acid receptor- $\gamma(24)$, a potential candidate gene for keratinization disorders. However, the characteristic histopathology of $\operatorname{EHK}(9,10)$, and the tissue specific expression of the defect limited to the suprabasilar keratinocytes, without evidence of systemic manifestations, suggest that this gene is less likely candidate for mutations in this family with EHK. Finally, although the EHK locus demonstrated co-segregation with COL2A1 locus without recombination, this gene is not a candidate gene since type II collagen is expressed exclusively in the cartilaginous tissues (25) which are not affected in EHK.
Recent studies have demonstrated that epidermolysis bullosa simplex, a heritable blistering disease, has been mapped either to chromosome $12 q$ or $17 q(6-8,12)$. It is conceivable, therefore, that either one of the constitutive subunits of the basal keratin heterodimers harbor mutations resulting in similar phenotypes. Based on this observation, it is possible that in other families with EHK, KRT 10 could be a candidate gene. In fact, recent publications have identified discrete point mutations both in KRT1 and KRT10 in several individuals with EHK (26-28). Several lines of evidence, including demonstrations that transfection of keratinocytes with a gene mutated by site-directed mutagenesis (27) or in vitro assembly of a mutated peptide (28) resulted in defective intermediate filament formation, suggested that these point mutations were responsible for the EHK phenotype. Consequently, these studies support our hypothesis that KRT1 is a candidate gene in the family examined in our study. Furthermore, demonstration of mutations in different regions of these keratin genes may explain the clinical variability noted in different families with EHK.

The demonstration of genetic linkage in this family now provides a means for accurate genetic counseling and prenatal diagnosis within this family. Development of polymerase chain reaction-based detection of restriction fragment length polymorphisms within or near the KRT1 locus could serve as a rapid means to elucidate the genotype in this family by amplification of the corresponding segment of DNA isolated from chorionic villus biopsy specimens, from periumbilical vein blood sample, or from fetal cells obtained through amniocentesis. This approach could replace the invasive procedure of fetal skin biopsy which was the only available means of diagnosis until now.

\section{Acknowledgments}

We thank the EHK family for their cooperation and their interest in this work. Dr. Mario DiLeonardo provided dermatopathological expertise. Drs. Eric Bernstein and Lidia Rudnicka assisted in photography. Dr. Douglas Kress, at the time a medical student at Jefferson Medical College, assisted in obtaining the blood samples. The skillful secretarial assistance of Debra Pawlicki is acknowledged.

This work was supported by grants PO1-AR38923 and T32AR7561 from the National Institutes of Health, and by the Dermatology Foundation.

\section{References}

1. Goldsmith, L. A. 1976. The ichthyoses. Prog. Med. Genet. 1:185-240.

2. Williams, M. S., and P. M. Elias. 1987. Disorders of cornification. In Dermatologic Clinics. Vol. 5, No. 1. J. C. Alper, editor. W. B. Saunders Co., Philadelphia, PA.

3. Ackerman, A. B. 1970. Histopathologic concept of epidermolytic hyperkeratosis. Arch. Dermatol. 102:253-159.

4. Uitto, J., E. A. Bauer, and A. N. Moshell. 1992. Symposium on epidermol- 
ysis bullosa: molecular biology and pathology of the cutaneous basement membrane zone. J. Invest. Dermatol. 98:391-395.

5. Uitto, J., and A. M. Christiano. 1992. Molecular genetics of the cutaneous basement membrane zone: perspectives on epidermolysis bullosa and other blistering skin diseases. J. Clin. Invest. 90:687-692.

6. Coulombe, P. A., M. E. Hutton, A. Letai, A., A. Hebert, A. P. Paller, and E. Fuchs. 1991. Point mutations in human keratin 14 genes of epidermolysis bullosa simplex patients: genetic and functional analyses. Cell. 66:1301-1311.

7. Epstein, E. H., Jr. 1992. Molecular genetics of epidermolysis bullosa. Science (Wash. DC). 256:799-804.

8. Lane, E. B., E. L. Rugg, H. Navsaria, I. M. Leigh, A. H. M. Haegerty, A Ishida-Yamamoto, and R. A. J. Eady. 1992. A mutation in the conserved helix termination peptide of keratin 5 in hereditary skin blistering. Nature (Lond.). 356:244-246.

9. Ishida-Yamamoto, A., J. A. McGrath, S. J. Chapman, I. M. Leigh, E. B. Lane, and R. A. J. Eady. 1991. Epidermolysis bullosa simplex (Dowling-Meara type) is a genetic disease characterized by an abnormal keratin-filament network involving keratins K5 and K14. J. Invest. Dermatol. 97:959-968.

10. Holbrook, K. A., B. A. Dale, V. P. Sybert, and R. W. Sagebiel. 1983. Epidermolytic hyperkeratosis: ultrastructure and biochemistry of skin and amniotic fluid cells from two affected fetuses and a newborn infant. J. Invest. Dermatol. 80:222-227.

11. Ishida-Yamamoto, A., I. M. Leigh, E. B. Lane, and R. A. J. Eady. 1992. Selective expression of the differentiation specific keratins $K 1$ and $K 10$ in the tonofilament clumps of bullous ichthyosis (epidermolytic hyperkeratosis). $J$. Invest. Dermatol. 99:19-26.

12. Ryynänen, M., R. G. Knowlton, and J. Uitto. 1991. Mapping of epidermolysis bullosa simplex mutation to chromosome 12. Am. J. Hum. Genet. 49:978-984.

13. Nakamura, Y., L. Ballard, P. O'Connell, M. Leppert, G. M. Lathrop, J. -M. Lalouel, and R. White. 1988. Isolation and mapping of a polymorphic DNA sequence pYNH15 on chromosome 12q (D12S17). Nucleic Acids Res. 16:779.

14. Weaver, E. J., and R. G. Knowlton. 1989. Characterization of a Hinf I polymorphism in the type II collagen gene (COL2A1). Cytogenet. Cell Genet. 54:1103.

15. Nakamura, Y., C. Martin, R. Myers, L. Ballard, M. Leppers, P. O'Connell, G. M. Lathrop, J.-M. Lalouel, and R. White. 1992. Isolation and mapping of a polymorphic DNA sequence (pCMM86) on chromosome 17q (D17S74). Nucleic Acids Res. 16:5223.

16. Ott, J. 1974. Estimation of the recombination fraction in human pedi- grees: efficient computation of the likelihood for human linkage studies. Am. J. Hum. Genet. 26:588-597.

17. Takahashi, E., T. Hori, P. O'Connell, M. Leppert, and R. White. 1990 R-banding and nonisotopic in situ hybridization: precise localization of the human type II collagen gene (COL2A1). Hum. Genet. 86:14-16.

18. Lessin, S. R., K. Huebner, M. Isobe, C. M. Croce, and P. M. Steinert. 1988. Chromosomal mapping of human keratin genes: evidence of non-linkage. J. Invest. Dermatol. 91:572-578.

19. Coulombe, P. A., M. E. Hutton, R. Vassar, and E. Fuchs. 1991. A function for keratins and a common thread among different types of epidermolysis bullosa simplex diseases. J. Cell Biol. 115:1661-1674.

20. Bonifas, J. M., A. L. Rothman, and E. Epstein. 1991. Linkage of epidermolysis bullosa simplex to probes in the region of keratin gene clusters on chromosomes 12q and 17q. J. Invest. Dermatol. 96:550 (Abstract).

21. McKinley-Grant, L. J., W. W. Idler, I. S. Bernstein, D. A. D. Parry, L. Cannizzaro, C. M. Croce, K. Huebner, S. R. Lessin, and P. M. Steinert. 1989. Characterization of a cDNA clone encoding human filaggrin and localization of the gene to chromosome region lq21. Proc. Natl. Acad. Sci. USA. 86:4848-4852.

22. Polakowska, R. R., R. L. Eddy, T. B. Shows, and L. A. Goldsmith. 1991. Epidermal type I transglutaminase ( $\mathrm{TgM} 1$ ) is assigned to human chromosome 14. Cytogenet. Cell. Genet. 56:105-107.

23. Compton, J. G., J. J. DiGiovanna, S. K. Santucci, K. S. Kearns, C. I. Amos, D. L. Abangan, B. P. Korge, O. W. McBride, P. M. Steinert, and S. J. Bale. 1992. Linkage of epidermolytic hyperkeratosis to the type II keratin gene cluster on chromosome 12q. Nature Genet. 1:301-305.

24. Mattei, M.-G., M. Riviere, A. Krust, S. Ingvarsson, B. Vennstrom, M. Q. Islam, G. Levan, P. Kautner, A. Zelent, and P. Chambon. 1991. Chromosomal assignment of retinoic acid receptor (RAR) genes in the human, mouse, and rat genomes. Genomics. 10:1061-1069.

25. Vuorio, E., and B. deCrombrugghe. 1990. The family of collagen genes. Annu. Rev. Biochem. 39:837-872.

26. Rothnagel, J. A., A. M. Dominey, L. D. Dempsey, M. A. Longley, D. A Greenhalgh, T. A. Gagne, M. Huber, E. Frenk, D. Hohl, and D. R. Roop. 1992. Mutations in the rod domains of keratins 1 and 10 in epidermolytic hyperkeratosis. Science (Wash. DC). 257:1128-1130.

27. Cheng, J., A. J. Syder, Q.-C. Yu, A. Letai, A. S. Paller, and E. Fuchs. 1992. The genetic basis of epidermolytic hyperkeratosis: a disorder of differentiationspecific epidermal keratin genes. Cell. 70:811-819.

28. Chipev, C. C., B. P. Korge, N. Markova, S. J. Bale, J. J. DiGiovanna, J. G. Compton, and P. M. Steinert. 1992. A leucine $\rightarrow$ proline mutation in the $H$ subdomain of keratin 1 causes epidermolytic hyperkeratosis. Cell. 70:821-828. 